\title{
Transparent Parallelization of Enrichment Operations in Geometric Modeling
}

\author{
Pierre Bourquat, Hakim Belhaouari@a ${ }^{a}$, Philippe Meseure, Valentin Gauthier and Agnès Arnould \\ University of Poitiers, CNRS, XLIM UMR 7252, 86000 Poitiers, France \\ \{hakim.belhaouari, philippe.meseure $\} @$ univ-poitiers.fr
}

\begin{abstract}
Keywords: Geometric Modeling, Parallelism, Parallel Construction of Virtual Objects, Transparently-Parallel Modeling Operation, Generalized Maps.

Abstract: $\quad$ This paper presents an approach to automatically and transparently parallelize algorithms to build 2D or 3D virtual objects in geometric modeling: In particular, we show that subdivision and Iterated Function System constructions can be parallelized, without any explicit parallelization study by their developer. These operations are described in the framework Jerboa, where each operation is expressed as a graph transformation and objects are topologically described using generalized maps. All transformations are handled by a generic engine that can handle structure changes in parallel. The obtained results allow any designer of virtual environments to rely on modern multi-core and multi-processor architectures to get faster constructions of complex objects without any skills on parallelism.
\end{abstract}

\section{INTRODUCTION}

Geometric modeling is usually based on a set of geometric operations that modify a given $2 \mathrm{D}$ or $3 \mathrm{D}$ object locally. During the building process, the zone where a local operation is applied must be precisely defined.

However, some operations involve an entire object, such as $2 \mathrm{D}$ or 3D refinement, volumetric extrusion, etc. as well as geometric operations used in Iterated Function Systems (Hutchinson, 1981). These constructions are often implemented as a succession of local operations. For instance, a 2D object can be refined by successively subdividing each of its faces. For large and complex objects, with many elements, this process can be quite time-consuming. For instance, building fifth iteration of a Menger sponge applied on fourth iteration requires about 50 seconds using Linear Cell Complex in $\mathrm{CGAL}^{1}$ on a i7-7820HQ $2.9 \mathrm{GHz}$

Considering modern multicore processors and multiprocessor architectures, the application of this process could be accelerated using parallelization. A naive approach mainly consists in parallelizing an operation by applying a local, initially sequential, algorithm at several places on the object at the same time. However, if topological consistency is wanted

\footnotetext{
a (iD) https://orcid.org/0000-0003-4454-7756

${ }^{1}$ https://www.cgal.org/
}

(finding shared vertices, edges, faces, etc.), this naive parallel approach fails at coping with the shared features efficiently. Indeed, they lead to concurrent accesses that heavily penalize performances due to critical sections or any other synchronous barrier introduced in the parallel program. Naturally, the topology of the object could be coped with in a ultimate phase, but this process (where any data is potentially shared) would lose the time benefits of the operation parallelization in itself. The only way to avoid such an ultimate phase is to find a numbering convention in the structure (for vertices, edges, faces, etc.), that implicitly contains adjacency relations. This numbering convention requires a dedicated study for each operation.

In this article, we present an automatic parallelization without human intervention of some geometric operations in order to build complex objects. These operations are called "enrichment operations" because they add new elements to an object (a more formal definition is given in the paper). These operations constitute the main steps that are required to transform a simple object into a more complex one.

Our method guarantees to represent correct adjacency relations between topological cells (vertices, edges, faces, volumes) even if these relations are intrinsically shared between cells processed during a transformation.

Moreover, parallelizing an operation usually starts 
from its sequential version and, as a subsequent step, consists in making it parallel. On the contrary, our approach aims at automatically parallelizing a given operation, without any intervention of its developer by precisely analyzing topology.

To meet this goal, we choose to rely on Jerboa, a freely-available modeling platform ${ }^{2}$. This tool provides us with an adapted framework, mainly consisting of a rule-based language for graph transformations that provides any transformation rule with static and dynamic checks. Jerboa is based on different engines that handle these rules to carry out geometric operations (Belhaouari et al., 2014). We found that these engines were well-suited to parallelism and could be enhanced and optimized in time for some global geometric transformations. More precisely, we propose an adapted rule engine that gives, automatically, a parallel implementation of geometric transformations based on parallel graph manipulations.

The paper is therefore organized as follows: After the presentation of related work in section 2, Jerboa is detailed in section 3. Our new parallel engine is then presented in section 4. Performance of this engine is detailed in section 5 followed by conclusion and future work.

\section{RELATED WORK}

Few geometric modeling algorithms have benefited from concurrent programming. Indeed, they are usually applied on a local area of an object and handle few vertices, edges, etc. However, some modeling operations deal with a consequent part or the totality of an object and can be accelerated using parallelization if the object to build is complex. This mainly concerns global mesh processing methods (Coupez et al., 2000) and, among these, subdivision algorithms. However, the parallelization is usually dedicated to a given subdivision method, for instance the Butterfly approach in (Padrón et al., 2002). A recent method has tried to gather several subdivision schemes into a unique algebra (Mlakar et al., 2019). This approach is efficient by focusing on the computation of new vertex positions but is dedicated to subdivision schemes only.

Some approaches only aim at producing on-the-fly refinement in parallel but not with the intent to store the final mesh (Nießner et al., 2012). On the contrary, other approaches deal with the complexity of the final mesh by distributing the memory requirements and

\footnotetext{
2 https://xlim-sic.labo.univ-poitiers.fr/ jerboa
}

manipulation between processing units. Theses approaches need to separate the manipulated mesh into clusters. This allows for the use of sequential algorithms inside a cluster but the management of cluster borders needs data exchanges between processing units (Damiand et al., 2018). Strategies have been proposed to limit these exchanges for specific subdivision schemes (Gargallo-Peiró et al., 2017). The partitioning of the manipulated mesh into clusters can lead to load balancing problem and a fine-grained approach can be interesting (Chen et al., 2013).

More generally, geometric algorithms are also used during construction in procedural modeling as well as in Iterated Function Systems (IFS) (Hutchinson, 1981). Procedural modeling can benefit from parallelization, but existing methods are usually restricted to linear structures (Lipp et al., 2010) and not surface or volumetric structured meshes.

Our goal is to allow for the building of topologically consistent complex objects using parallelization to speed-up construction. Our method can also be seen as a parallelized approach to build IFS, for nonlinear structures, on modern platforms by relying on the multicore and multiprocessor architectures.

We consider that the main contributions of our method are: (1) the generation of complex, quasimanifold surface or volumetric objects, with a noticeable speed-up, (2) the automatic and transparent parallelization of any building process based on enrichment operations using a fined-grained approach, (3) the guaranty that the adjacency relations are correctly computed and updated during a building process.

Our parallelization approach has been made possible due to the specific properties of Jerboa, that is, the inner structure to represent topology and the way geometric transformations are handled. More precisely, parallelization is made possible thanks to Jerboa's rule syntax. As a consequence, our approach heavily depends on its strategy to cope with geometric, and more exactly topological transformations. This means that our method cannot be applied directly on classical geometric modeling API, since transformation are not handled the same way as in Jerboa (using a formal approach that mainly copies, pastes and modifies graph patterns). The next section is thus dedicated to the presentation of this tool.

\section{GENERAL PRESENTATION OF JERBOA}

Jerboa is a rapid prototyping platform dedicated to topology-based geometric modeling. It allows a designer to quickly specify one or more geometric oper- 
ations on $2 \mathrm{D}$ or 3D models. It heavily relies on graph transformations theory and uses a dedicated graphic language to design transformation rules via an editor. Not much code has to be written to create an operation, since only the way to handle and update any information embedded in the model (such as new position of vertices) after a transformation must be given. The runtime execution of an operation is done using an engine that interprets a called rule and applies changes on a selected object. To understand how Jerboa can be a valuable tool to parallelize geometric operations, it is mandatory to understand the inner structure and the language used by transformation rules. These features are thoroughly described in (Belhaouari et al., 2014), but, in this section, we recall elements that help understanding how parallelization can be applied.

\subsection{Inner Topological Structure}

The inner structure of Jerboa relies on a generalized combinatorial map (G-map). This structure is inspired from half-edges (Campagna et al., 1998) but is generalized for any dimension, and allows for the representation of non-oriented manifolds (Lienhardt, 1994). Thus, a G-map may be represented as an unoriented graph, where nodes are basic elements called darts and $\operatorname{arcs}^{3}$ are labeled by dimension (called $\alpha_{d}$ for a relation associated with dimension $d$ ). Indeed, arcs always aim at linking two entities of a given dimension explicitly known by the value of their label.

Figure 1 presents the decomposition of a 3D complex object along decreasing dimensions. Two volumes are linked along a "dimension 3" relation $\left(\alpha_{3}\right)$ (Fig. 1a). Then, one volume is decomposed into faces linked by a "dimension 2" relation $\left(\alpha_{2}\right)$ (Fig. 1b). Successive edges on a face are linked by a "dimension 1" relation $\left(\alpha_{1}\right)$ (Fig. 1c). Finally, one edge is composed of two darts linked by a "dimension 0" relation $\left(\alpha_{0}\right)$ (Fig. 1d). The topology of the initial object is described in Figure 1e where all geometrical information (embeddings) has been deleted.

Some constraints must be verified to ensure the consistency of a G-map. Typically, each dart must be connected to other darts with one and only one relation of each dimension, from 0 to $n$ where $n$ is the dimension of the object to which it belongs to. Concerning dangling condition, a dart must be linked to another dart or to itself. If a dart is linked by $\alpha_{i}$ to itself, it is said to be $\alpha_{i}$-free. On Figure 1e, some darts do not have $\alpha_{3}$ links (these links are not repre-

\footnotetext{
${ }^{3}$ Dedicated jargon to avoid ambiguity between "edge" from graph theory and "edge" from geometry, i.e. segment between two vertices. Our "arcs" are not oriented.
}

sented): They correspond to faces with no adjacent volume. Another constraint deals with the so called "manifold condition". Following $\alpha_{i}$ and then $\alpha_{j}$ or $\alpha_{j}$ and then $\alpha_{i}$ from a starting dart must lead to the same dart, when $i+2 \leq j$. All these conditions are verified within Jerboa environment, most of them in a static way at design stage, so that no inconsistent structure can be generated.

With this formalism, topological cells (vertices, edges, etc.) are defined as a subgraph with a particular set of relations, defined as their labels. For instance, from a given dart $d$, in 3D, the subgraph $\left\langle\alpha_{0}, \alpha_{2}, \alpha_{3}\right\rangle$ (d) denotes the edge including $d$. $\left\langle\alpha_{1}, \alpha_{2}, \alpha_{3}\right\rangle$ (d) denotes the vertex that includes $d$. $\left\langle\alpha_{0}, \alpha_{1}, \alpha_{2}\right\rangle$ (d) denotes the incident volume. $\left\langle\alpha_{0}, \alpha_{1}, \alpha_{2}, \alpha_{3}\right\rangle$ (d) stands for the whole connected component to which $d$ belongs and so on. In addition, G-maps support supplementary subgraphs, that are called orbits that are not necessarily topological cells, and we call "orbit type" the list of links to follow to get all the darts of an orbit. For instance, it is possible to use the corner of a face with the orbit type $\left\langle\alpha_{1}\right\rangle$ or make a difference between a $2 \mathrm{D}$ edge (orbit type $\left\langle\alpha_{0}, \alpha_{2}\right\rangle$ ) between two faces (possibly belonging to the same volume) and a $3 \mathrm{D}$ edge (orbit type $\left\langle\alpha_{0}, \alpha_{2}, \alpha_{3}\right\rangle$ ) shared by several volumes.

This feature is heavily used to formalize embeddings. In Jerboa, when additional data must be embedded in a G-map, an orbit type must be associated with each data for Jerboa to be able to check consistency after topological changes. Every orbit of a type associated with a given data should embed a value. Thus, since Jerboa ensures this property, it detects where a developer of an operation forgets to add a computation of a value for a new orbit that appears after a transformation when this orbit type should embed a data. Jerboa also ensures that every dart of an orbit that embeds a data share the same value.

\subsection{Definition of an Operation}

In Jerboa, graph transformations are used to modify a G-map. This approach comes originally from formal methods and allows Jerboa to preserve consistency directly from the description of an operation. This section is dedicated to the graphical language that a developer uses to describe an operation, in the form of a transformation rule. The following section presents how rules are interpreted and executed by a unique engine. Rules are divided into two parts: the left hand side which indicates the pattern where the operation should be applied and the right hand side which explains transformations from the left pattern.

Figure 2a illustrates the well-known Catmull- 


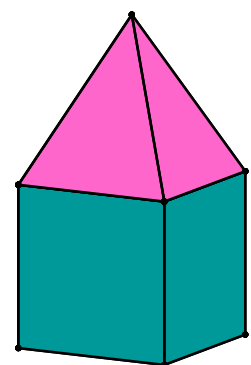

(a) Volume decomposition

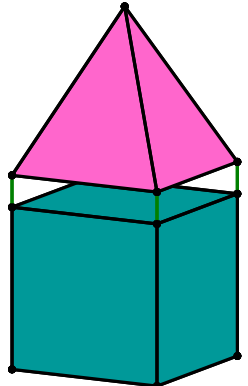

(b) Face decomposition

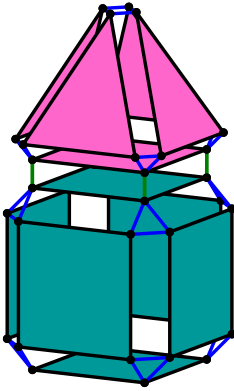

(c) Edge decomposition

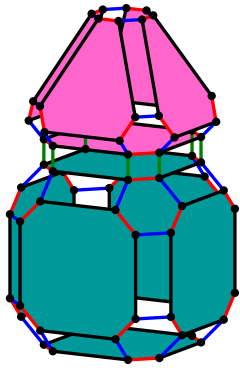

(d) Vertex decomposition

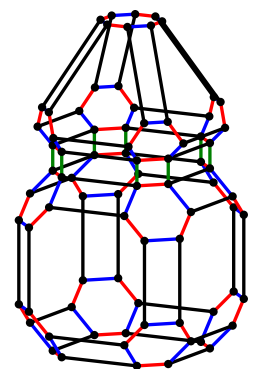

(e) Final topology

Figure 1: Object decomposition into a G-map. $\alpha_{3}$ are represented in green, $\alpha_{2}$ in blue whereas $\alpha_{1}$ are red. $\alpha_{0}$ are black.

Clark subdivision operation in Jerboa (Catmull and Clark, 1978). The left pattern has a unique node which is labeled with an orbit to represent a specific element. Here, the orbit type $\left\langle\alpha_{0}, \alpha_{1}, \alpha_{2}\right\rangle$ is shown, that means that the subdivision must be applied on a set of connected faces (that can be a unique volume if the surface is closed). A looping arc labeled $\alpha_{3}$ indicates that this set of faces must be free along dimension 3, that is to say: No face of the set is connected to a volume. Obviously we could make a more general operation that subdivides any face of several connected volumes, but it would make the example more complex and we want to keep it as simple as possible by not coping with $\alpha_{3}$ links. The right hand side has four nodes and topological relations. The most left node represents the original set of faces where edges have been deleted ( $\alpha_{0}$ removed) so that another node can connect to this one. The two center nodes represent a new geometric vertex at the midpoint of each edge of the initial object. More precisely, these nodes represent face corners, due to the $\alpha_{1}$ link between them. The most right node contributes to the new vertex positioned at the center of each initial face.

The application of such a rule is heavily based on a folding mechanism (Ehrig et al., 2006) on each dart of the initial object. To better understand this mechanism, let us apply it to a simple quad linked by one of its edge to a triangle. Bottom of Figure 2 shows a first step of the construction. First, the node of the left part of the rule is identified with all the darts of the object where the operation is applied, that is, in our example, the eight darts of the quad and the six darts of the triangle (Fig. 2b). This node is kept ( $\mathrm{n} 0$ ) and duplicated three times (n1, n2 and n3) in the right part of the rule. In practice, this means that three new groups of fourteen darts are created (represented, in Figure 2c, with a color code corresponding to a node of the rule). Some relations are automatically created between these darts, by renaming (changing the di-

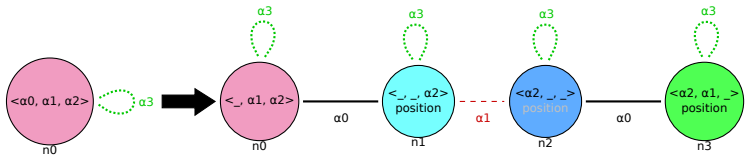

(a) Rule representation

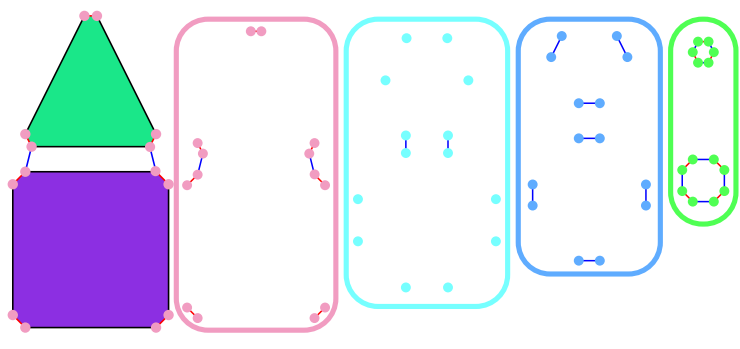

(b) Node identification

(c) Node duplication

Figure 2: Catmull-clark subdivision operation in Jerboa.

mension of) already-existing relations in the original node. These are called "implicit" links and are written inside each node. For instance, when duplicating the initial left node to create node called $n 3$, all $\alpha_{0}$ links from the initial quad are kept but renamed into $\alpha_{2}$ to link the newly-created darts. $\alpha_{1}$ links are kept as in the initial quad. $\alpha_{2}$ links are discarded. The initial node can also be modified, here $\alpha_{0}$ are deleted, as previously mentioned.

Explicit links (corresponding to an arc in a rule) are then built between the new darts to complete the construction, as this can be seen in Figure $3 b$ (with the same color code than in Fig. 2a). For instance, a dart in node $n 1$ created as a duplication of a dart in node $\mathrm{n} 0$ is linked to this dart with an $\alpha_{0}$ link, as stated in the operation of Figure 2a. Figure 3c illustrates the folding mechanism where Jerboa rule can be recognized for each of the 14 darts of the original node. Note that all links, for all dimensions, are handled by the rule for every node, so for all the darts they include 


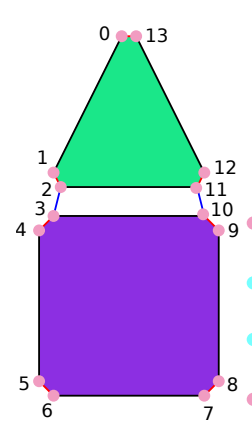

(a) Initial

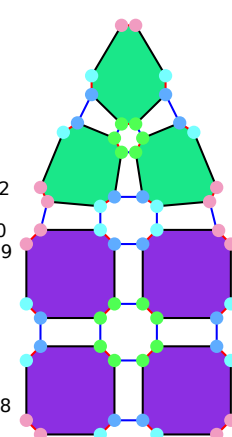

(b) Final object

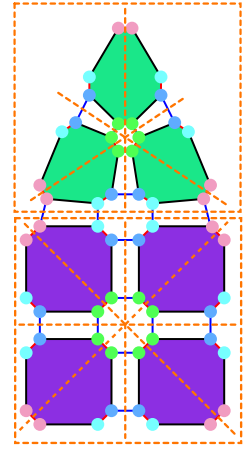

(c) Folding mechanism
Figure 3: Result of the application of Jerboa Catmull-Clark rule and focus on the application of this transformation rule on each of the 14 darts of the initial mesh (in pink).

(newly-created or modified). If not, such a rule would be considered as inconsistent (which is verified at the design of the rule) and could not be applied.

The rule in Figure $2 \mathrm{a}$ also points out that the position of the vertices inserted in the midpoint of edges and faces must be computed, whereas positions of initial vertices do not change (smoothing is ignored in this example).

It must be emphasized that Jerboa does not carry out an operation like other geometric modeling environments. Indeed, Catmull-Clark is usually implemented by first introducing a midpoint on each edge, creating a vertex at the midpoint of each initial face before erasing this latter, creating adjacent edges for every midpoint vertex and creating new faces based on subdivided and newly created edges. On the contrary, Jerboa rather consider an operation in terms of node identification, duplication, label renaming for arcs, etc. Jerboa always relies on the same process, whatever the desired operation written in its graphic language. This process is handled by a generic, that is "operation-independent", engine that is described in the following section and allows for a completely transparent parallelization of any operation.

\subsection{Jerboa Engine}

The formalism presented in previous section offers a significant advantage: A unique algorithm, so called "Jerboa Engine" handles any Jerboa rule describing an operation. This section describes the main steps of this engine, but focuses on the steps used by enrichment operations and ignores the steps only required by other kinds of operation (even if they are effectively handled by Jerboa's general purpose engine).

The first step is the identification of the left pattern of an executed rule. More precisely, this step aims at mapping the left, symbolic, node with a set of all darts belonging to the orbit type written inside this node. This step also checks folding properties around orbits. In our example, it identifies the full set of connected faces (or the unique volume) given as parameter to the operation.

The second step consists in checking developer preconditions with the found left pattern. It allows, for instance, a user to check embedding values after the engine checks topology aspect.

The third step is a complex step where the right pattern of the applies rule is intensively employed to cope only with topological aspects. First, the engine creates, for each node in the right pattern, as many new darts as darts identified during the first step. For instance in the Catmull-Clark operation, it creates for each existing dart of the identified node, three new darts (identified by node $n 1, n 2$ and n3). Then, the engine rewrites topology information, that is create links for each dart, in accordance with the operation. It proceeds in two steps. First, it handles implicit arcs. Each label inside nodes of the right pattern is analyzed. If a link type is not marked as '.' in a node's label, it is reproduced between the set of darts associated to the considered node according to the links between darts identified with the left pattern. Each link can be renamed before it is reproduced, that is, it is possible to change the dimension of the link. Second, the engine analyzes explicit arcs between any two connected nodes in the right pattern to link each dart associated to one of these nodes to its related neighbor dart on the other node.

The fourth step is dedicated to embedding values. For every embedding of the object, the engine must take an inventory of all orbits of its associated type to handle embedding values for each of them. The engine makes a difference between values that have to be computed and already-existing values, defined on initial orbits and that only need to be spread when these orbits are modified. For computed values, the designer has to provide a computing code (in a dedicated language).

\section{PARALLEL TRANSFORMATION ENGINE}

The generic algorithm to handle operations shown in previous section gives the opportunity to design a parallel process of any construction operation. First, we have to analyze the different stages of Jerboa Engine to find potential parallelization issues and deadlocks. 


\subsection{Jerboa Engine Analysis}

The steps of the Jerboa Engine seem simple at first sight, but they hide many difficulties and implementation issues. The most significant difficulties appear during embedding evaluation and refer to orbit manipulation.

The engine computes embedding values in the initial object space because, this way, computations may reuse values already present in this object, such as orbits, neighbor darts or initial embedding values. However, the engine must determine where computations must be called, so it also manipulates the final object to determine every orbit of any orbit type that embeds information. A similar problem appears when an already-existent embedding value, associated with a given orbit type, must be spread from an initial dart to newly-created darts belonging to the same orbit. The engine handles this issue by determining every orbit (of a given type, the one associated to a given embedding), that is, partitioning the set of darts into equivalence classes of darts belonging to the same orbit, and distribute an embedding value to all the darts constituting it.

All these issues lead us to classify operations with respect to their topological properties. Currently, we identify three classes: update operations that do not induce topology changes but only imply modification of embedding values, enrichment operations which have only one node as left pattern and increase the number of darts without deletion, and other operations. This article focuses on enrichment operations, since they are used to make an object more complex (subdivision, triangulation, extrusion, etc.), and both update and enrichment operations can benefit from the same optimization approach. On the contrary, considering the last class of operations, where deletions might be required, consequent treatments to satisfy consistency of topology and embedded values must be used and heavily complicate parallelization. Moreover, update and enrichment operations generate less concurrent access issues contrary to other kinds of rules and are more likely to benefit from parallelism. We decided to let this third class of rules as future work.

Now, let us consider the different steps of Jerboa Engine (see section 3.3). In enrichment operations, the left pattern is reduced to one node identified with one orbit type that defines the zone where the enrichment operation is applied.

The second step resorts to user code (to manage embedding values), so a dedicated automatic code analysis is required to parallelize this step. This is not our purpose, so we let these steps sequential and use them as barriers to synchronize other steps. The third step, that is the one that interprets the right part of the rule, offers several parallelization opportunities.

\subsection{Parallelized Engine for Enrichment Operations}

At first, the engine checks if the rule given as input is compatible with parallelism, that is, if it is an update or an enrichment rule: (1) There is a unique node in the left pattern of the rule, (2) this unique node must have a full label orbit (no symbol bottom ',' can be used), (3) the left node is preserved on the right pattern (then no deletion occurs). Our approach also assumes that computation of embedding values are concurrent-safe.

Note that, to ensure consistency constraints of enrichment operations, the orbit associated with the left node usually corresponds to a complete connected component, in enrichment operations.

\subsubsection{Parallelizing Topological Transformations}

When the engine starts the application of an operation, it determines, first, all darts which represent the unique node of the left pattern by walking through the orbit shown in its label. With this list of darts (called left darts), our engine computes an indexed structure to organize topological transformations efficiently. Contrary to Jerboa orginal data structures, such an indexed, local, structure is common and it allows for the use of parallel patterns ( $\mathrm{McCool}$ et al., 2012). It appears as a matrix that gives, for all indexed darts, the index of the darts that are 0-linked with them in a first line, those that are 1-linked in a second line, and so on for every link. This matrix is first used to represent the initial structure, as can be seen in Table 1. Its size is the number of left darts and is called "left adjacency matrix". It is filled in the first step of the algorithm, during the gathering of all left darts. A similar structure is also allocated to represent the transformed structure, and is called "right adjacency matrix". Since left darts are duplicated for each node in the right pattern, the size of this right matrix is the product of the number of left darts and the number of nodes in the right pattern. These matrices must ensure non-orientation constraints: If a dart $d$ is linked to a dart $e$ for a given dimension, then it must be ensured that $e$ is linked to $d$ for the same dimension. The following algorithms guarantee this property. Note that, concerning update operations, left and right matrices are the same.

The index of each dart is determined this way: If $l$ is the number of left darts, the darts of the right pre- 
Table 1: Left matrix of object in Figure 3a.

\begin{tabular}{|c|c|c|c|c|c|c|c|c|c|c|c|c|c|c|}
\hline ID & 0 & 1 & 2 & 3 & 4 & 5 & 6 & 7 & 8 & 9 & 10 & 11 & 12 & 13 \\
\hline$\alpha_{0}$ & 1 & 0 & 11 & 10 & 5 & 4 & 7 & 6 & 9 & 8 & 3 & 2 & 13 & 12 \\
\hline$\alpha_{1}$ & 13 & 2 & 1 & 4 & 3 & 6 & 5 & 8 & 7 & 10 & 9 & 12 & 11 & 0 \\
\hline$x$ & 0 & 1 & 3 & 2 & 4 & 5 & 6 & 7 & 8 & 9 & 11 & 10 & 12 & 13 \\
\hline
\end{tabular}

served node are numbered from 0 to $l-1$, as the same index stands for darts of the left pattern (index used in the left matrix) and their image in the preserved node (index used in the right matrix). Darts created for the first new node in the right pattern are numbered $l$ to $2 l-1$, and so on. More generally, if an initial dart $k$ is duplicated in right node $h$, the index of the copy is $h \times l+k$ ( $h=0$ corresponds to the preserved node). At this point, no more search or walk-through are carried out outside of this range of darts since any node used by the operation is indexed and corresponds to an entry in the structure.

The computation of topology consists in filling the different lines of the right matrix, by first coping with implicit links and then with explicit ones.

Concerning implicit links, for a given right node, our engine inspects the word of its orbit label. Each word means a mapping of links of a given dimension $i$ to all the links of a possible other dimension $j$ in the initial structure (renaming mechanism). Our method just copies the line $j$ of the left matrix into the line $i$ in the right matrix at indices corresponding to the concerned node. An offset of $h \times l$ is applied to find destination columns but must also be applied on each copied value, since the darts concerned by these implicit links are not indexed from 0 to $l-1$ but from $h \times l$ to $(h+1) \times l-1$ if $h$ is the current processed node. Parallelism is carried out over each word in the orbit label of each right node and for each copied link.

Concerning explicit arcs, parallelism is handled over each explicit arc present in the rule and each left darts. Remember that an explicit arc of dimension $e$ between two nodes $a$ and $b$ binds any dart $n_{a}$ in $a$ to its corresponding dart $n_{b}$ in $b$. Corresponding dart means that $n_{a}$ and $n_{b}$ come from the same left dart $k$. Hence, we have $n_{a}=a \times l+k$ and $n_{b}=b \times l+k$. It is therefore possible to directly fill, in parallel, the line $e$ for columns $n_{a}$ and $n_{b}$ for each $k$ between 0 and $l-1$.

\subsubsection{Parallelizing Embeddings Management}

The next stage handles embeddings. This step includes two parts: determination of where the computation must be called in the final topology and effective computation in the initial topology. This duality is solved easily since the right matrix presented in previous section represents the modified object whereas the left matrix and the current G-map of the object still represent the initial state. Here again, parallelism is possible for both aspects.

The determination of where the computation must occur consists in finding a unique representative dart for each orbit of a given type in order to avoid redundant computation of the same value among darts of the same orbit. We propose the so-called "islet algorithm" that computes representative darts of all orbits of the same type simultaneously. This algorithm takes a set of darts and an orbit type as input, and it gives as output, for each dart, the representative dart of the orbit to which it belongs for the specified orbit type and a list of the representative darts, and consequently, the list of orbits. The algorithm consists in tightening incrementally each $\alpha_{i}$ link, where $\alpha_{i}$ belongs to the selected orbit type. The PRAM complexity is $O(m)$ where $m$ is the size of the biggest orbit of the zone concerned with the embedding to compute or propagate.

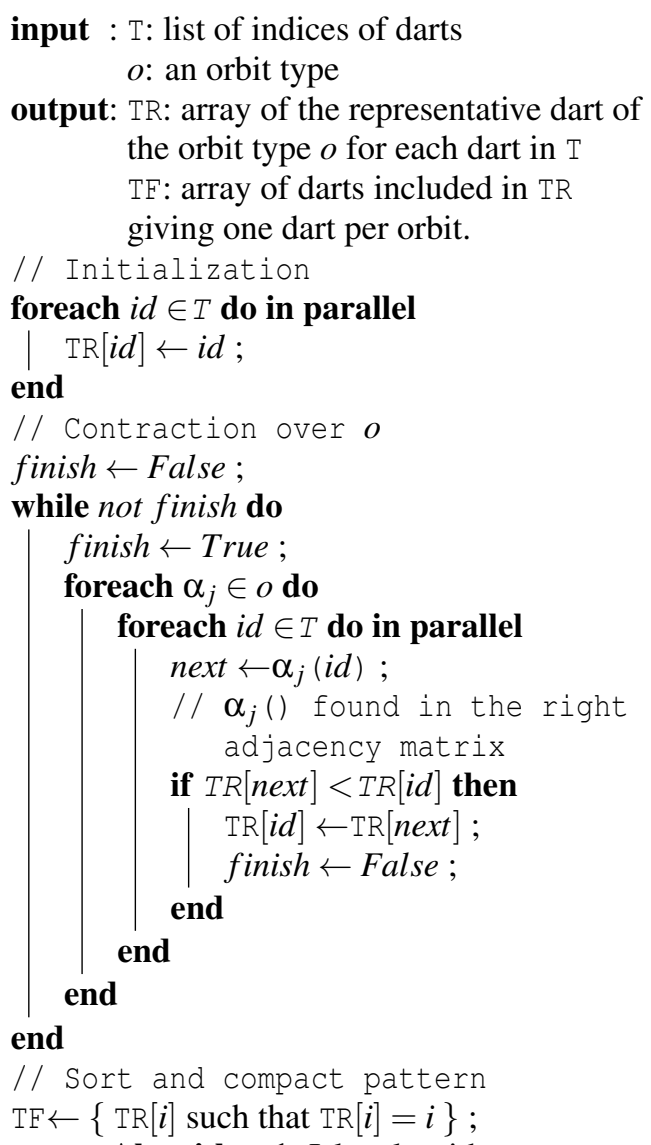

Algorithm 1: Islet algorithm.

Algorithm 1 details the different steps. First, two shared structures are initialized: TR that stores the representative dart selected by each dart in its orbit for the selected orbit type and $\mathrm{TF}$ a list of representative darts (initially empty). Each dart selects itself as the 


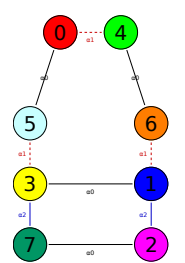

(a)

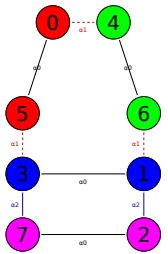

(b)

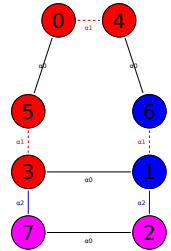

(c)

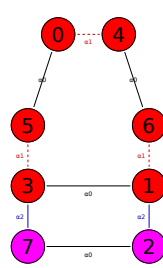

(d)
Figure 4: Execution of the Islet algorithm for orbit type $\left\langle\alpha_{0}, \alpha_{1}\right\rangle$.

representative dart of its orbit, so $\operatorname{TR}[i] \leftarrow i$. Then, a loop starts. Each step deals with all the $\alpha_{i}$ links from the input orbit type. The algorithm spreads the representative dart with the minimal index along each $\alpha_{i}$. In practice, for each dart $d$ and its neighbor $e$ linked by $\alpha_{i}$, the algorithm selects the dart with the minimal index among their representative darts $\operatorname{TR}[d]$ and $\operatorname{TR}[e]$ and update the value of the greater one. This loop is iterated until no contraction occurs after trying each link $\alpha_{i}$ of the selected orbit type. Finally, a classic compact pattern removes redundancy in TR to keep only one dart per orbit in $\mathrm{TF}$.

Figure 4 shows an execution of this algorithm on an object to find all representative darts for orbit type $\left\langle\alpha_{0}, \alpha_{1}\right\rangle$ (face orbit). The label inside each dart (circle) always gives its index. A color is associated with each index and, along the algorithm, shows which representative dart is selected by each dart. Initially, no contraction has been applied and each dart selects itself as its representative dart for the orbit. This is why, in Figure 4a, each dart has a different color (dart 0 is red, 1 is blue, and so on). The algorithm contracts alternatively $\alpha 0$ and $\alpha 1$ in order to spread representative darts. Figure $4 \mathrm{~b}$ shows the result after a contraction over $\alpha 0$. Here, dart 5 takes the color of dart 0 because they are linked by $\alpha_{0}$ and the index of dart 0 is lower than dart 5. For the same reason, dart 6 takes the color of dart 4, and so on. Figure $4 \mathrm{c}$ displays the next contraction over $\alpha 1$. Now, darts 3 and 4 also select dart 0 respectively from dart 5 and dart 0 . At this point, the algorithm has determined that darts $0,3,4$ and 5 belong to the same face orbit. Figure $4 d$ shows the final result where dart 0 has been elected by all the darts of the above face. Dart 2 has been elected for the below face. No more contraction occurs, the algorithm stops. The object is composed of two faces (the red and the pink ones).

Once Islet algorithm has built TR and TF, TF is first used to compute the values for the embedding value associated to each orbit. TR is then used to spread this value so that all the darts of an orbit share the same embedding value. Finally, all the topological modifications computed in the local adjacency matrix are copied (in parallel) to update the global G-map.

\section{RESULTS}

This section presents different applications and time performance for different operations. It must be emphasized that these results rely on strictly the same parallel Jerboa engine, which handles a dedicated rule for each example presented in this section. The engine is compiled once for any rules independently on operation and it could be used for any other enrichment operation that is expressed in Jerboa formalism. The proposed examples aim at showing the versatility of our approach. We first study several subdivision operations for surface meshes, and then show an example of a 3D IFS. For each case, Jerboa rules are shown. Since Jerboa has been first developed in Java, our parallel engine has also been implemented in this language, in order to validate the approach for parallelization. Our measures have been carried out on a general purpose machine, with a processor i77820HQ 2.9GHz with 8 (hyperthreaded) cores, 32GB of RAM and JDK 11.

\subsection{Subdivision surfaces}

Subdivision surfaces offer good examples to present our transparent parallelism. There are different classes of subdivision schemes depending on subdivided elements and face kinds (triangular or quad). We can cite those that split faces into sub-faces and those that split vertices into multiple vertices. The Loop (Loop, 2002) and Catmull-Clark (Catmull and Clark, 1978) subdivisions divide faces into subfaces. The former is dedicated to triangular meshes and the latter to quad meshes. We have also implemented Doo-Sabin subdivision (Doo and Sabin, 1978). This one splits vertices of faces instead of face directly. Figures 2a, 5a and 6a present implementation of such operations in Jerboa. The two first selected subdivisions produce regular patterns: Using Loop's approach, triangles are subdivided into four sub-triangles (Figs. 5b-5d) and in Catmull-Clark, quads become four sub-quads (Fig. 3b). On the contrary, Doo-Sabin subdivision depends on adjacent faces around vertices (Figs. 6b-6d). By the way, it can be remarked that the right pattern of these operations include four nodes, but with different relations linking them. Thus, the role of each node depends on the operation: For instance, nodes $n 1, n 2$ and $n 3$ in Loop's rule (Fig. 5a) represent a same vertex. In Doo-Sabin's rule (Fig. 6a), all nodes represent the same vertex which is expected since this scheme works solely on 


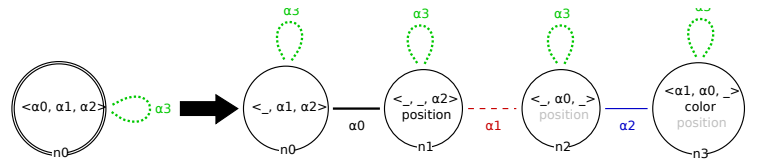

(a) Jerboa rule

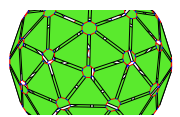

(b)

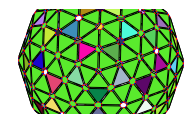

(c)

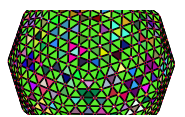

(d)
Figure 5: Loop subdivision rule and some applications.

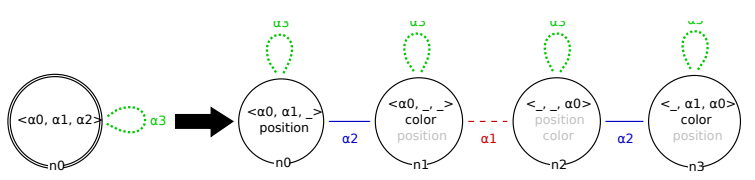

(a) Jerboa rule

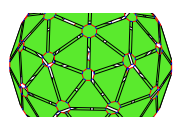

(b)

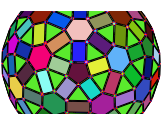

(c)

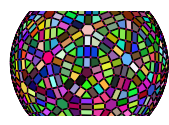

(d)
Figure 6: Doo-Sabin subdivision rule and some applications.

vertices.

Figure 7 shows time execution of each subidivision with respect to the number of iterations of various tools/libraries.

We choose open source libraries such as OpenSubDiv (Polson, 2013) which offers various types of execution, MeshLab (Cignoni et al., 2008) a wellknown tool of the community, OpenFlipper (Möbius and Kobbelt, 2012) a GUI of OpenMesh, a topologybased modeler, and CGAL with polyhedron (The CGAL Project, 2019) and linear cell complexes (LCC) (Damiand and Teillaud, 2014) that are based on combinatorial maps. All these tools/libraries are developed in $\mathrm{C}++$ in a classic way, with much dedicated optimization, carefully studied by their developers and specialized in the targeted operation. Since we propose an automatic and general parallelization, the same for any enrichment operation, it clearly can not offer the same performance. Moreover, our results are also harmed by Java, the implementation language chosen by Jerboa, and, more precisely, by (recentlyimplemented) Java Streams and Java Garbage Collector that attempts to free memory when many darts are allocated (note that all experiments exceed millions of darts, it peaks about 30 million darts on Loop experiment). However, our parallel engine gives better results than Jerboa sequential default engine after some iterations when overhead time fades into data charge. Concretely, at the end of the experiment, the paral- lel engine is from two to three times faster than the default one.

This experiment shows many elements that interfere with results.First, the overhead time generated by parallelism may influence performance badly: As an example, OpenSubDiv (which obtains the best results of this experiment) has better result with a sequential computation (about $10 \mathrm{~ms}$ ) than parallel one (about $13 \mathrm{~ms}$ ). Second, dedicated optimization of the code influences drastically performance: CGAL LCC has good performance for Loop operation whereas performance for Catmull-Clark is catastrophic after the fourth iteration: It seems that this operation has been less optimized. This last remark speaks in favour of our transparent parallelism approach where a unique engine is developed for all operations instead of giving the same parallelization effort for each kind of operation. To strengthen this last argument, we notice that some tools offer the ability to apply multiple iterations in a single step or one after the other (as Jerboa does). This functionality provided by some tools implies, here again, dedicated, specifically-studied, optimization to benefit from parallelism. For instance, OpenFlipper obtains 8 Loop subdivision at once in $4061.285 \mathrm{~ms}$ and one by one $7089.825 \mathrm{~ms}$. The same is observed for MeshLab where iterations in one step take $226 \mathrm{~ms}$ and the application of several iterations $555 \mathrm{~ms}$, thus a clear difference indicates a global optimization in this treatment.

Table 2: Detailed execution time of the 8th iteration with our parallel engine.

\begin{tabular}{|l|c|c|c|}
\hline Steps & Doo-Sabin & Loop & Catmull-Clark \\
\hline \hline $\begin{array}{l}\text { Left pattern identification and } \\
\text { checks }\end{array}$ & $17.30 \%$ & $18.89 \%$ & $14.06 \%$ \\
\hline $\begin{array}{l}\text { Matrix building and topology up- } \\
\text { date }\end{array}$ & $7.84 \%$ & $8.71 \%$ & $6.8 \%$ \\
\hline Creation of new darts & $33.51 \%$ & $36.91 \%$ & $24.25 \%$ \\
\hline $\begin{array}{l}\text { Embedding computation and con- } \\
\text { sistency checks }\end{array}$ & $41.34 \%$ & $35.49 \%$ & $54.58 \%$ \\
\hline
\end{tabular}

Finally, Table 2 presents an extract of detailed execution time of each step of our parallel engine (cf. Section 3.3). The identification of the left pattern and initial checks take less than $20 \%$ for any operation. We notice that the computation of new topology is really fast and take less than $9 \%$. The real bottleneck that has been confirmed previously comes from the allocation of many darts inside the JVM that takes around third of the whole iteration time, then computation of embeddings takes what remains (around $40 \%$ ). This last fact could be worse without islet algorithm as we originally processed the embedding step the same way as the identification of the left pattern (so we would get similar performance). Instead of 


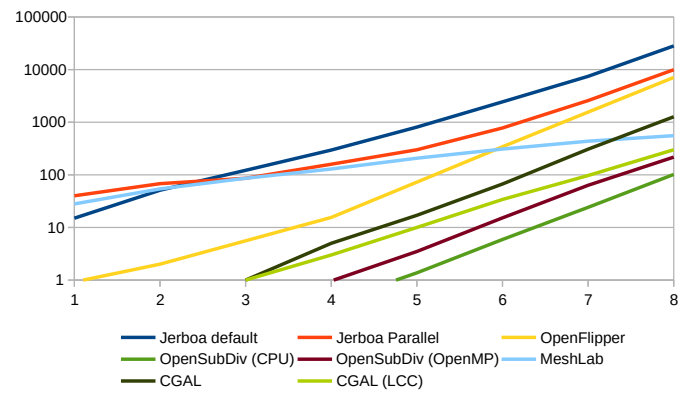

(a) Execution time for Loop

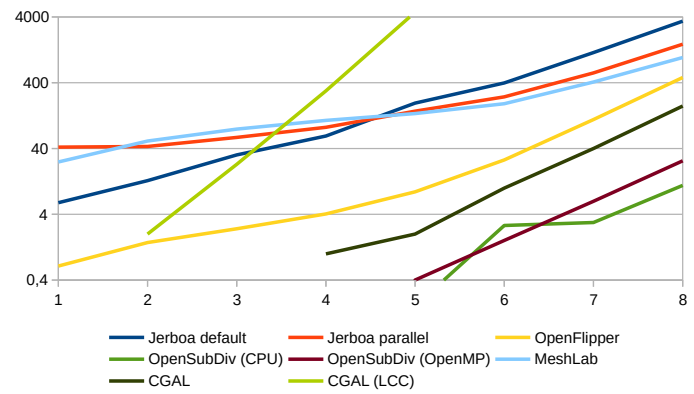

(b) Execution time for Catmull-Clark

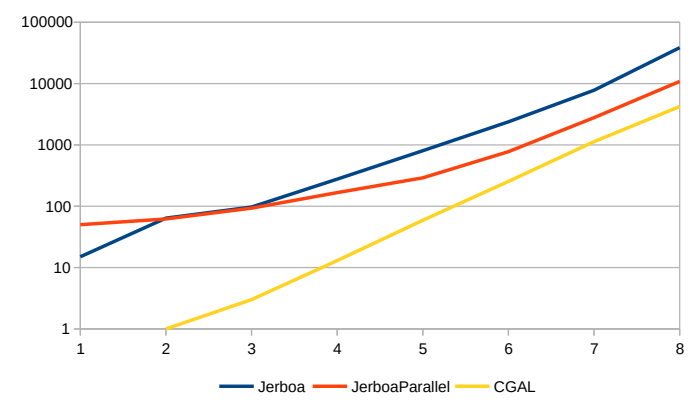

(c) Execution time for Doo-Sabin

Figure 7: Execution time for subdivision operations.

computing each orbit one after the other, islet algorithm gives all the orbits of a given type, which explains the computation gain. Note that embedding step is applied for each embedding (two embeddings in our experiment: vertex position and face color) and for each embedded value, the computation provided by the user is called without any optimization.

\section{$5.23 D$ IFS}

The Menger sponge operation is a good 3D fractal for our purpose. Figure 9a presents the adaptation of this operation. This rule follows all specificity of enrichment operation and it is applied on a whole connected component. The right hand side is complex and it has twenty nodes (static checks of manifold condition have been helpful to design such a rule). This operation is commonly applied on a cube for topological purposes (Figs. 9b-9d) but we can directly apply this rule on any object if it respects presented properties. Figures $9 \mathrm{e}-9 \mathrm{~g}$ present successive application of (the same) Menger operation on a prism.

Figure 8 shows execution time of successive applications of this operation on a cube. The obtained results are in accordance with those in 2D. Thanks to parallelization, it is shown that the number of nodes in the right hand side of a rule does not influence performance. The yellow line represents execution time

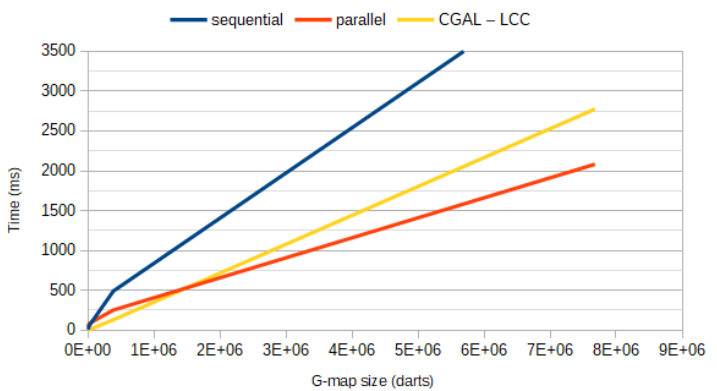

Figure 8: Execution time for Menger sponge rule.

of a C++ implementation of this computation developed in the CGAL LCC framework. We remark that the parallel engine is best for the last iteration despite the JVM behind it, whereas CGAL is programmed in $\mathrm{C}++$.

\section{CONCLUSION AND FUTURE WORK}

We have proposed an approach to parallelize some operations in geometric modeling. More precisely, we focused on so-called enrichment operations, that is operations that make an object more complex and, 


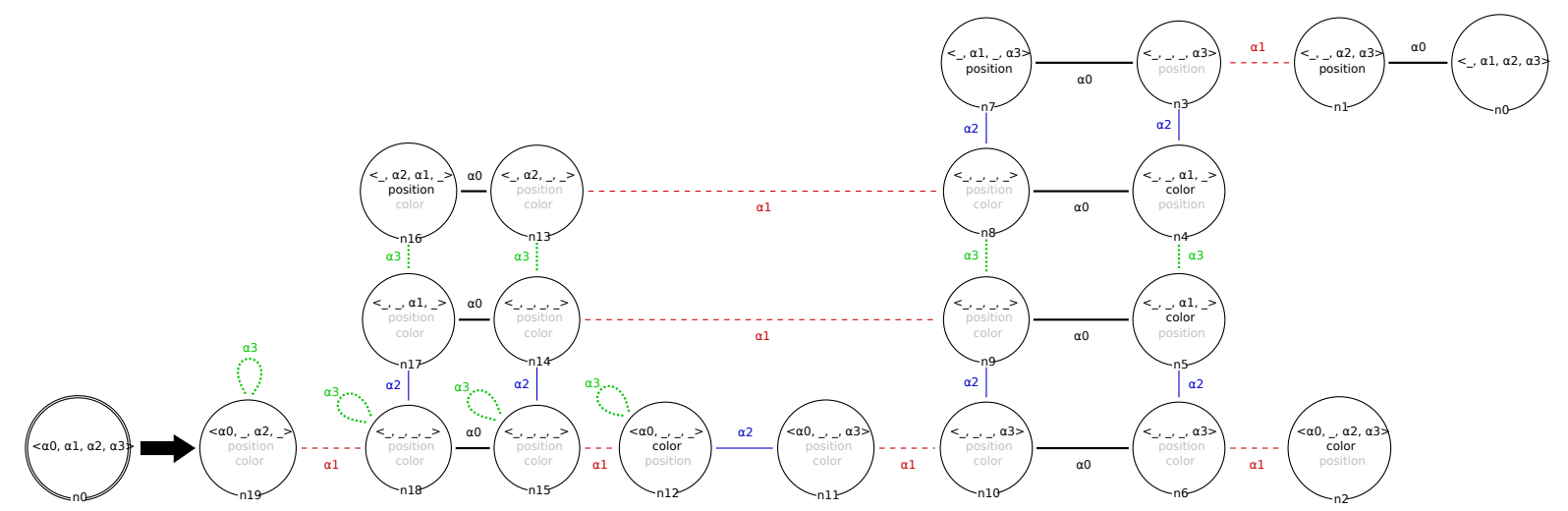

(a) Jerboa rule

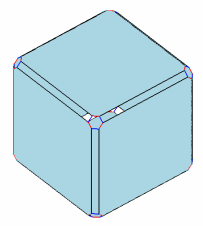

(b)

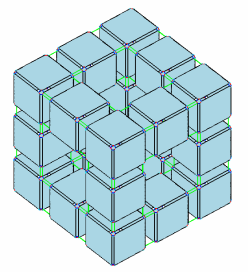

(c)

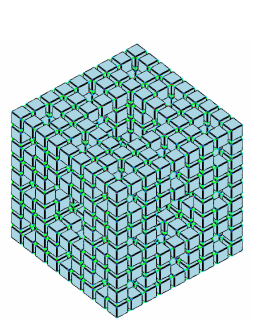

(d)

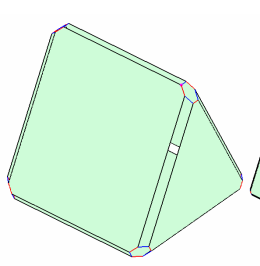

(e)

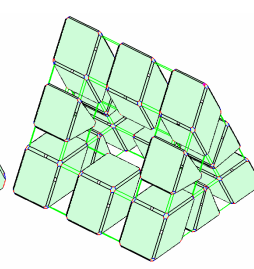

(f)

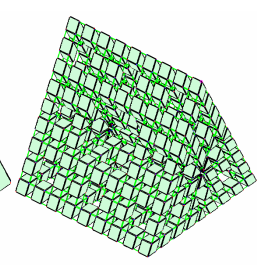

(g)

Figure 9: Menger operation and some applications

more generally, operations that aim at building objects. We relied on Jerboa structure and transformation method that is based on graph transformations. Any enrichment operation must be expressed as a Jerboa rule, that is handled by a generic engine that has been parallelized. This approach guarantees that any operation that can be expressed as an enrichment operation can benefit from the parallelization without the need for a dedicated study, that is, specificallystudied parallelization of all the steps of the operation as a classical geometric modeling approach would require.

Parallelization patterns have been identified in Jerboa's generic Engine and two steps have been parallelized: the topological transformation stage and the management of embeddings. The used topological structure is a key point of our approach since it offers a fine-grained decomposition of the object that allows us to deal with adjacency links of different dimension separately.

The obtained results are clearly encouraging (x2 or $\mathrm{x} 3$ speed-up) and the optimization is satisfactory. A conversion of this engine to $\mathrm{C}++$ will probably give us more efficiency when using multicore architectures than Java Streams and will, hopefully, allow us to compete with dedicated optimized methods. However, one point still needs attention: Only two steps of the process have been parallelized. The first stage of the process, that consists in filtering the part of the object that is transformed and building the local matrices that the parallel engine needs is still sequential. Its parallelization requires to be able to walk through a topological structure (a graph) in parallel but such a walk-through requires critical sections that heavily penalize performance. Another possible track to get better performance is to port the engine presented in this paper on GPU, since the matrices that it relies on can be easily transferred to graphics memory. Finally, the paper focuses on update and enrichment rules. Some other, non local, rules, including several input nodes and/or darts deletion, could be interesting to parallelize as well.

\section{REFERENCES}

Belhaouari, H., Arnould, A., Gall, P. L., and Bellet, T. (2014). Jerboa: A graph transformation library for topology-based geometric modeling. In 7th International Conference on Graph Transformation 2014, volume 8571 of Lecture Notes in Computer Science, pages 269-284.

Campagna, S., Kobbelt, L., and Seidel, H.-P. (1998). Directed edges-A scalable representation for triangle meshes. Journal of Graphics Tools, 3(4):1-11.

Catmull, E. and Clark, J. (1978). Recursively generated 
B-spline surfaces on arbitrary topological meshes. Computer-Aided Design, 10(6):350-355.

Chen, J., Zhao, D., Zheng, Y., Huang, Z., and Zheng, J. (2013). Fine-Grained parallel algorithm for unstructured surface mesh generation. In International Meshing Roundtable.

Cignoni, P., Callieri, M., Corsini, M., Dellepiane, M., Ganovelli, F., and Ranzuglia, G. (2008). MeshLab: an Open-Source Mesh Processing Tool. In Eurographics Italian Chapter Conference.

Coupez, T., Digonnet, H., and Ducloux, R. (2000). Parallel meshing and remeshing. Applied Mathematical Modelling, 25(2):153-175.

Damiand, G., Gonzalez-Lorenzo, A., Zara, F., and Dupont, F. (2018). Distributed combinatorial maps for parallel mesh processing. Algorithms, 11(8).

Damiand, G. and Teillaud, M. (2014). A Generic Implementation of $\mathrm{dD}$ Combinatorial Maps in CGAL. In Proc. of 23rd International Meshing Roundtable (IMR), volume 82 of Procedia Engineering, pages 4658.

Doo, D. and Sabin, M. (1978). Behaviour of recursive division surfaces near extraordinary points. ComputerAided Design, 10(6):356 - 360.

Ehrig, H., Ehrig, K., Prange, U., and Taentzer, G. (2006). Fundamentals of algebraic graph transformation. EATCS Series "Monographs in Theoretical Computer Science". Springer-Verlag.

Gargallo-Peiró, A., Houzeaux, G., and Roca, X. (2017). Subdividing triangular and quadrilateral meshes in parallel to approximate curved geometries. In 26th International Meshing Roundtable.

Hutchinson, J. (1981). Fractals and self similarity. Indiana University Mathematical Journal, 30(5):713-747.

Lienhardt, P. (1994). N-dimensional generalized combinatorial maps and cellular quasimanifolds. International Journal of Computational Geometry and Applications, 4(3):275-324.

Lipp, M., Wonka, P., and Wimmer, M. (2010). Parallel generation of multiple L-Systems. Computer and Graphics, 34(5):585-593.

Loop, C. (2002). Smooth ternary subdivision of triangle meshes. Curve and surface fitting, 10(6):3-6.

McCool, M., Robison, A. D., and Reinders, J. (2012). Structured parallel programming. Elsevier/Morgan Kaufmann, Amsterdam Boston.

Mlakar, D., Winter, M., Seidel, H.-P., Steinberger, M., and Zayer, R. (2019). AlSub: fully parallel and modular subdivision. https://arxiv.org/abs/1809.06047.

Möbius, J. and Kobbelt, L. (2012). Openflipper: An open source geometry processing and rendering framework. In Curves and Surfaces, volume 6920 of Lecture Notes in Computer Science, pages 488-500.

Nießner, M., Loop, C., Meyer, M., and DeRose, T. (2012). Feature-adaptive GPU rendering of Catmull-Clark subdivision surfaces. ACM Transactions on Graphics, 31(1).

Padrón, E. J., Amor, M., Bóo, M., and Doallo, R. (2002). Efficient parallel implementations for surface subdivi- sion. In Fourth Eurographics Workshop on Parallel Graphics and Visualization.

Polson, B. (2013). Opensubdiv from research to industry adoption. In ACM SIGGRAPH 2013 Courses.

The CGAL Project (2019). CGAL User and Reference Manual. CGAL Editorial Board, 5.0 edition. 